\title{
Die erste Apologie Justins
}

Ein Versuch die Bittschrift Justins in ihrer ursprünglichen Form
herzustellen.

Von J. A. Cramer in 's Gravenbage.

I.

Als mir vor zwei Jahren die freundliche Gelegenheit geboten wurde, in dieser Zeitschrift meine Abhandlung über die Logosstellen in Justins Apologien zu veröffentlichen, teilte ich als mein Ergebnis mit, daß uns in Justins Apologien zwei Schriften vorlägen, eine von Justin und eine von einem alexandrinischen Juden-Christen, Schriften, die später zu einer Apologie umgearbeitet wurden. Es dünkte mich der einzige Weg zur Auflösung der bisher ungelösten Schwierigkeiten.

Eine weitere kritische Prüfung hat mir nicht nur das bisherige Resultat bestätigt, sondern mir auch mehr und mehr den Gedanken nahe gelegt, eine Wiederherstellung der ursprünglichen Form zu versuchen. Mit welchen Schwierigkeiten ein solcher Versuch verbunden ist, weiß ich wohl. Dennoch scheint er mir nicht vergeblich zu sein.

$\mathrm{Da}$ die Apologie (I und II), wie sie uns jetzt vorliegt, unmöglich das Werk eines einzigen Schriftstellers sein kann, darüber ist kein Wort mehr $z \mathrm{u}$ verlieren. Veil ${ }^{\mathrm{x}}$ gibt eine Übersicht über den Inhalt und den Gedankengang der justinischen Rechtfertigung des Christentums. ${ }^{2}$ Diese Úbersicht mit ihren Abteilungen und Unterabteilungen, wozu nicht nur die Ziffern I, II und III, und die Buchstaben A und B, sondern auch die Ziffern I, 2 und 3 und die Buchstaben a, b, c, d, ja sogar die griechischen $\alpha, \beta, \gamma, \delta$ in stets neuen Combinationen verwendet werden, während für mehrere „Episoden" gar kein Obdach zu finden ist, liefert

- I Justinus des Philosophen und Märtyrers Rechtfertigung des Christentums (Apologie I und II), eingeleitet, verdeutscht und erläutert. Straßburg, 1894.

2 S. 58-64. 
den besten Beweis für meine Behauptung und zeugt mehr für die Gewandtheit des Verfassers dieser Übersicht, als für die Geschicklichkeit des griechischen $\cdot$ Apologeten.

Man hat es sich überhaupt mit der Sache ziemlich leicht gemacht. Widersprüche seien nichts Wunderbares; sie kämen fast bei jedem Schriftsteller vor! Als ob jedermann, der Kritik übte, das nicht wübte! Wenn man jedoch überall Nähte und Ritze sieht, die man niemals gesucht, sondern $\mathrm{zu}$ sehen genötigt worden ist, braucht man doch nicht auf Grund einer handschriftlichen Überlieferung, um deren Text es, wie Harnack, Veil u. a. nachgewiesen haben, ${ }^{x}$ so schlimm steht, sich abschrecken zu lassen, die Apologien dort zu zerschneiden, wo die Ritzen von selbst den Weg dazu zeigen.

Die Sache würde sehr viel schwieriger sein, wenn die Aussagen des Eusebius uns keine Handhaben darböten und wenn zweitens der Inhalt der einzelnen Abschnitte uns nicht mannigfach den Weg zeigte. Eusebius ist nicht nur in textkritischer Hinsicht größeres Gewicht beizulegen als gewöhnlich geschieht, sondern auch in geschichtlicher Beziehung sollten seine Berichte genauer in betracht gezogen werden. Schon in meinen obenerwähnten Aufsatz habe ich darauf hingewiesen, daß die Beschaffenheit der Apologien es wahrscheinlich mache, daß die Stücke, die Eusebius aus Apol. II als aus I stammend zitiert, auch wirklich in der ersten Apologie gestanden haben. ${ }^{2}$

Die Stücke, die Eusebius aus Apol. II zitiert, sind: die bekannte Geschichte des Christenprozesses unter Urbicus (II, 2) der Anfang des

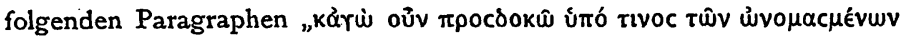

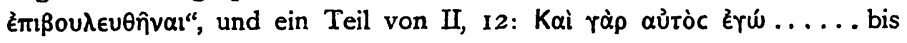
poveuӨncó $\mu \in v o v$, wo Justin von seiner eigenen Bekehrung zum Christentum spricht.

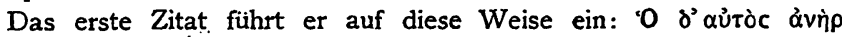

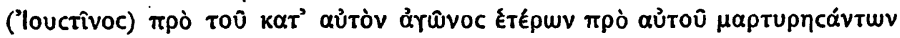

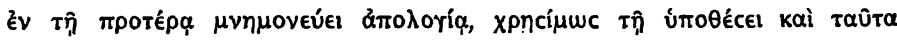

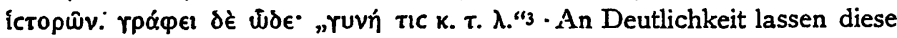
Worte nichts zu wünschen übrig. Die Bemerkung, Justin habe die Apologien in anderer Reihenfolge gehabt als wir, verliert alle Berechtigung, wenn man erwägt, daß Eusebius das Zitat aus Apol. II, 12 in

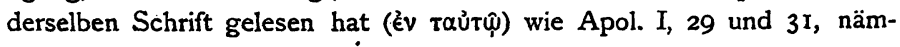

I Harnack, Die Überlieferung der griechischen Apologeten des zweiten Jahrhunderts (Texte u. Unters. I, 1883, S. 1-300) S. 135.

2S. 302. : 3 Euseb. H. e. IV, 17. I. Laemmer, 25. 


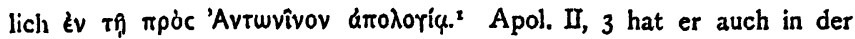
crsten Apologie gelesen. Nachdem er die Geschichte des Prozesses unter Urbicus erzählt, fährt er fort: toútoic $\delta$ 'louctîvoc elkótwe kal

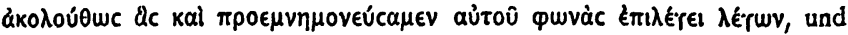
dann folgt der Anfang von Apol. II, 3. Also Apol. II, 2, 3, 12 hat Eusebius in derselben Schrift gelesen als Apol. I, 29 und $31 .^{2}$

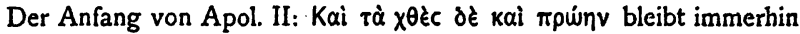
sehr auffallend. Mit Sylburg ist v. Otto der Meinung, Justinum indignatione commotum ex abrupto sic contexuisse exordium. ${ }^{3}$ Späterhin spürt man aber von dieser Entrüstung wenig oder nichts mehr. Apol. II, 5 erzählt uns den Abfall der Engel; II, 6 bezeugt, daß man Gott, dem Herrn, keinen Namen beilegen kann; II, 7 finden wir die Behauptung, die Menschen handeln nicht, wie die Stoiker lehren, infolge eines Verhängnisses, sondern Gott habe sie mit freiem Willen erschaffen; II, 9 werden wir gewarnt, die Lehre von der zukünftigen Feuerstrafe nicht für Großsprecherei $z u$ halten, weil es einen Unterschied gebe zwischen Tugend und Laster, wie sehr man sich auch bemühe, die Verschiedenheit der menschlichen Gebräuche dem gegenüber geltend zu machen; II, Io haben wir wieder ein Stückchen Logoslehre und II, II gibt uns sogar die Erzählung von Herakles auf dem Scheidewege. Und wir fragen, wo der Mann geblieben sei, der "indignatione commotus" an seine Apologie eine Nachschrift hinzufügt.

Apol. II, I gehört zur ersten Apologie und damit auch Apol. II, 2

Euseb. H. e. IV, 8, 3 .

2 Was Euseb. H. e. IV, I7 in der ersten Apologie liest, liest er H. e. IV, 16, I

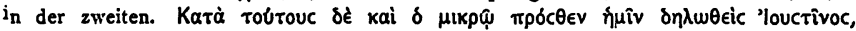

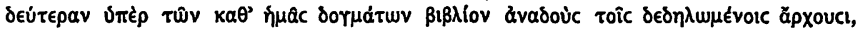

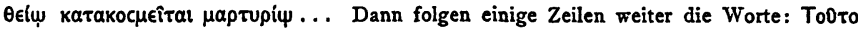

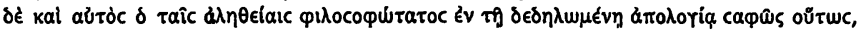

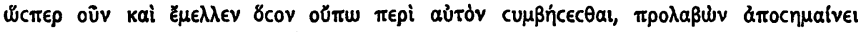

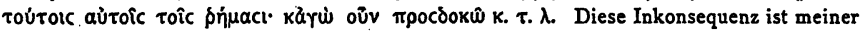
Meinung nach nicht anders zu erklären als durch die Annahme, Eusebius habe nur durch Überlieferung von zwei Apologien Justins gewußt, in Wahrheit nur eine gehabt. Er glaubt es sei Crescens gelungen, Justin den Märtyrertod zu bereiten (H. e. IV, 16), nicht sehr lange nachdem er seine 2 weite Apologie geschrieben habe. Aber wenn er schon i n der ersten Apologie die Vermutung ausspricht von Crescens getötet zu werden, wie kann er dann noch Zeit gefunden haben eine zweite Apologie zu schreiben? Er rettet sich aus der Verlegenheit, indem er den Bericht über Crescens anführt, als stünde er in der zweiten Apologie. Diese zweite Apologie hat Eusebius niemals gesehen. Er kannte nur eine Apologie, die, wie sich später zeigen' wird, sicherlich nicht mit unseren Apol. I und II identisch gewesen ist. Was Veil, a. W. S. XXIII, x bemerkt, ist völlig ung enügend.

3 Ed. III opp. Just. 1876, p. 194, annot. 2. 
und 3. Es liegt auf der Hand, daß die drei unechten Briefe, die Briefe Hadrians ${ }^{x}$, Antoninus und Marc Aurels hinter einander an die Apologie hinzugefugt sind. In der handschriftlichen Überlieferung ist die Reihenfolge: Apol. II, Apol. I, und dann die drei Reskripte. Veil meint, daß die ursprüngliche Reihenfolge diese war: Apol. I, Apol. II, während später die drei unechten Briefe angehängt worden sind. Mit Recht redet er dann von einer "seltsamen" Voranstellung von Apol. II." - Wie kann man das später als Apol. II bezeichnete Stück mitten aus dem $\mathrm{Zu}$ sammenhang heraus genommen und der Apol. I vorangestellt haben? Soll es bloßer Zufall gewesen sein und, wie Veil sagt, die alte Bucheinteilung mit der Übertragung der Schrift auf Pergamentblätter verloren-" gegangen sein?3

Es kann sein! Niemand wird auf diese Frage eine genügende Antwort geben können. Welche Handschrift dem Codex Parisinus zu Grunde gelegen hat, oder aus welchem Grund die verschiedenen Stücke auf diese Weise geordnet sind, ist schwerlich auszumachen. Es genügt zu wissen, daß Apol. I und II als eine Schrift zusammengehören und ferner, daß konservative Kritiker wie Veil doch die Möglichkeit zugeben, daß mit der Übertragung der Bittschrift Justins auf Pergamentblätter die alte Einteilung verloren gegangen sein kann. Hat vielleicht der Abschreiber des Codex Parisinus oder der Abschreiber der ihm zu Grunde liegenden Handschrift mehrere solche Pergamentblätter in Unordnung vor sich gehabt und beim Abschreiben Ordnung zu schaffen gesucht? Jedenfalls brauchen wir uns bei dem Versuch, die Apologie Justins in ihrer ursprünglichen Form herzustellen, nicht allzu peinlich um die handschriftliche Anordnung zu kümmern.

Fangen wir mit Apol. II, I an: Der Text ist ziemlich verdorben,

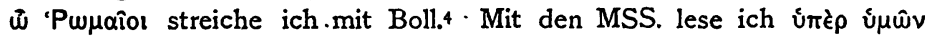
statt útrè I, 2 folgen zu lassen. Justin warnt Apol. I, 2 die Kaiser, nicht in unvernünftiger Leidenschaft sich selbst das Urteil zu sprechen. Er. und seine Mitchristen'können von niemandem etwas Böses leiden. Sie sind

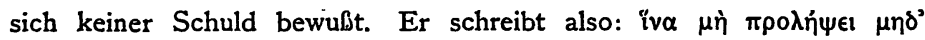

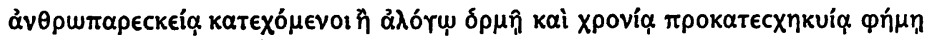

I Vgl. Keim, Rom und das Christentum, 1881, S. 559 und meine Abhandlung in Theol. Studiën, 189I, Bl. 422-426. Veil, a. a. O. S. 137-142.

2 A. a. O. S. 137.

3 A. a. O. S. XXVHI.

Zeitschr. f. d. hist. Theol., I842, III, S. 27. Auch Veil, a. a. O. S. 114. 


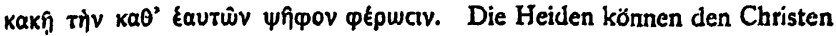
das Leben nehmen, schaden aber können sie ihnen nicht.

Warum schreibt Justin denn seine Apologie? Er tut es ihretwegen. Err könnte schweigen. Das aber, was unter Urbicus vorgekommen ist

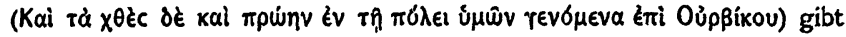
ihm Veranlassung zu ihrem Besten zu schreiben (i்tę $\dot{u} \mu \omega \hat{v})$. Die Worte

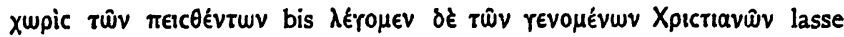

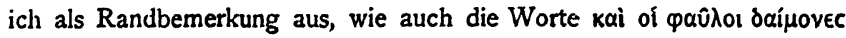

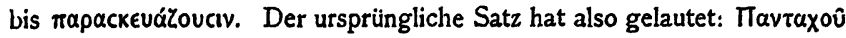

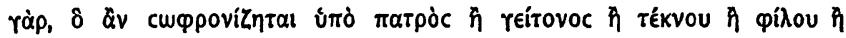

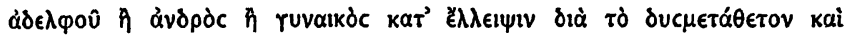

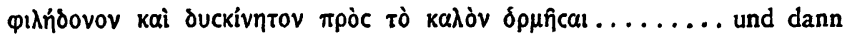

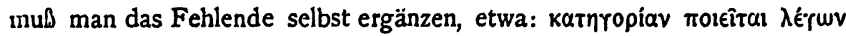

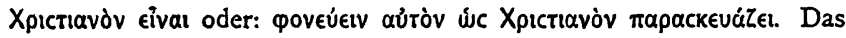

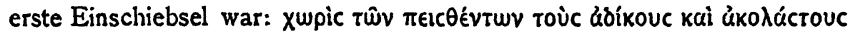

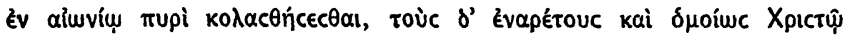

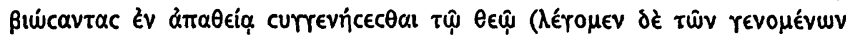

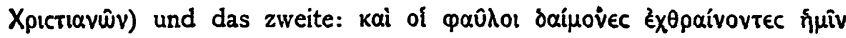

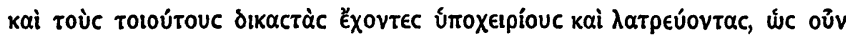

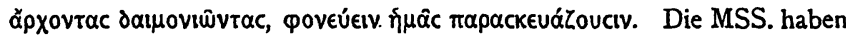
vor der zweiten Einschaltung eine Lücke. v. Otto sagt ${ }^{x}$ : non quod quidquam desit ad sententiae iuncturam sed quod deesse aliquid videretur. Er nennt sogar den Zusammenhang wie auch den Schlub des Satzes: aptissinus! Im Gegenteil ist aus dem holperichten Satze kein

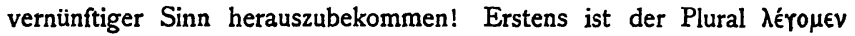

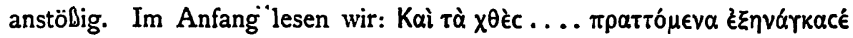

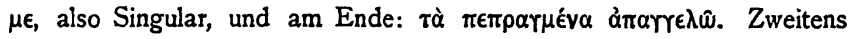

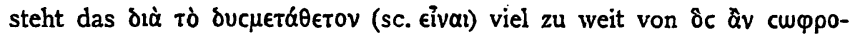
vízntal entfernt und kann auch nachher nicht mit einem Plural ( $\pi \alpha \rho \alpha-$ ckeválouciv) verbunden werden: Dieser Plural soll veranlabt sein durch

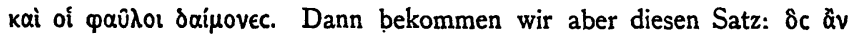

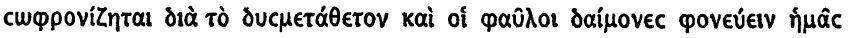
rapackeuáZouciv. Wenn das Griechisch ist, dann hat es mit allen Regeln der Syntax ein Ende! Drittens hat ôc ầ kein Prädikat. Viertens kommt das spätgriechische Wort Évópetoc ${ }^{2}$ oder évapétwc nur in unechten Stücken in der Apologie vor. 3 Fünftens ist die Bemerkung, daß

1 Opp. Just. ed. II, I, I, p. 196 ann. II.

2 Es kommt nur bei Herodian (Ende 2. Jahrh.) und bei Späteren vor (Pape).

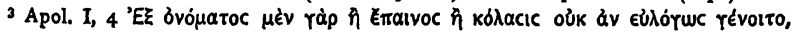


man überall den Tod der Christen sucht, nur diejenigen ausgenommen, welche Christen geworden sind, völlig sinnlos. $\mathrm{DaB}$ die Christeri nicht den Tod der Christen suchen, steht doch außer Zweifel. Sechstens lehrt Justin nirgends, daß die Tugendhaften zur leidensfreien Vereinigung mit Gott gelangen. Apol. I, 8 sagt er, dab diejenigen, welche freudig zum

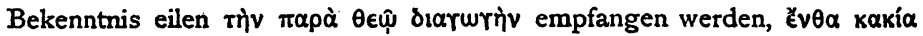
oủk àvtıiutrê. Veil übersetzt: wo das Böse keine Pein mehr schafft. Ich übersetze: wo das Böse keine Macht mehr übt, wo es keinen Widerstand mehr leistet. Von åđá $\theta \epsilon i \alpha$ ist hier nicht die Rede. Und was ist denin $\alpha \dot{\pi} \alpha \dot{\theta} \in \epsilon \iota \alpha$ ? Stumpfsinn oder. Unempfindlichkeit und, bei den Stoikern, Leidenschaftslosigkeit. ${ }^{2}$ Die Bedeutung: Leidensfreiheit habe ich nirgends angetroffen. Und endlich verrät die Dämonenepisode dieselbe Hand,

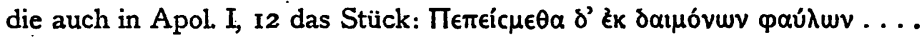
$\delta \ddot{\pi} \epsilon \rho$ $\theta \in \circ \hat{U}$ ěprov écti eingeschaltet hat. In meiner Abhandlung über die Logosstellen bei Justin habe ich $z u$ beweisen versucht, daß die Stellen, welche von den Dämonen handeln, mehr als verdächtig seien. ${ }^{3}$ Apol. I, I wo der Zusammenhang durch die Einschaltung verloren gegangen ist, bestätigt diese Meinung vollends. Ich lese also:

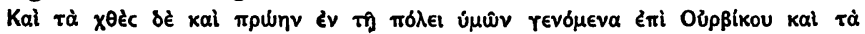

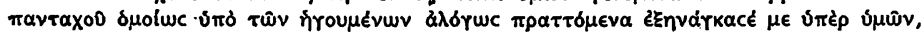

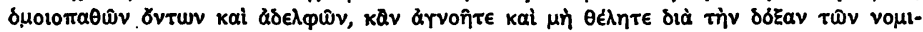

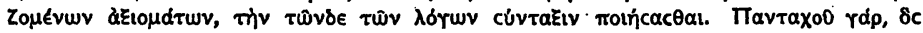

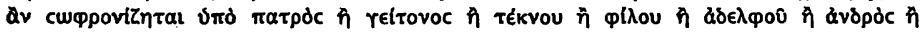

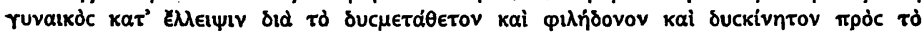

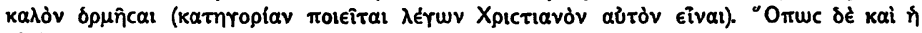

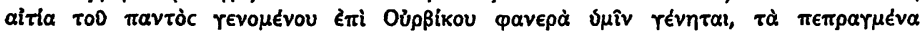
anarrelw.

Was Apol. II, 2 betrifft, das fast gänzlich in den MSS fehlt, tilge

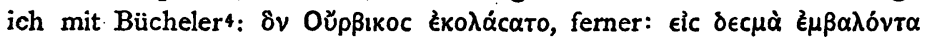

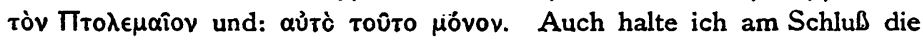

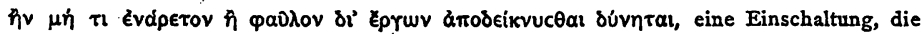

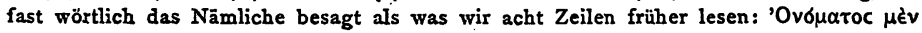

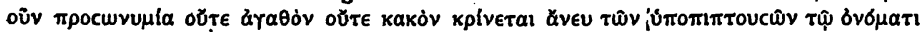
$\pi \rho d \xi_{\varepsilon w V}$. Apol. I, 2I, welches ganzes Stück unecht ist. Apol. I, I2 ist zwar von

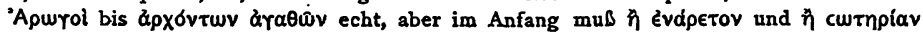
getilgt werden. Hagen (Zeitschr. f. Phil. u. kath. Theol., 65, 1848, S. 48) hat gefühlt, daß Evdpetov hier nicht am Platze steht. Ist hier doch nicht von Bösen und Guten, von Strafe und Belohnung, sondern nur von Übeltätern und Strafe die Rede. Er liest

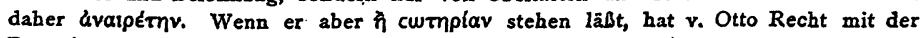
Bemerkung: non respicit quae sequuntur (1. c. pag. 36, annot. 2).

I A. 2. O. S. 5 .

2 Apol. I, 25, das nicht justinisch ist, wird Gott dima日nc genannt.

3 A. a. O. S. 312, 327, 330. 4 Rhein. Mus. XXXV, 1880, S. 286. 


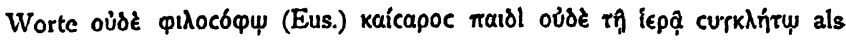
aus der verdorbenen Aufschrift geflossen für unecht. Dab ein leiden-

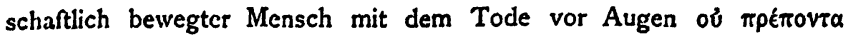

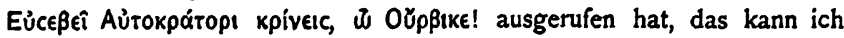

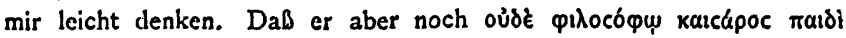

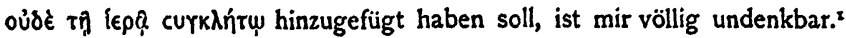

Auf Apol. II, 2 lasse ich nach Eusebius unmittelbar den Abschnitt über Crescens folgen. Ich sage: nach Eusebius; denn ich bleibe trotz der Einwendungen von Harnack ${ }^{2}$ und Veil ${ }^{3}$ noch immer der Meinung, daß die Bemerkung Hist. eccl. IV, 17, 13 nichts andres bedeuten kann, als dab im Exemplar des Eusebius der Abschnitt über Crescens unmittelbar auf Apol. II, 2 folgte. Ich sage dieses nicht auf Grund des

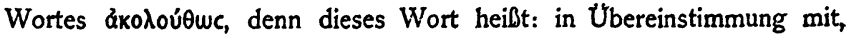
und darum übersetze ich mit Veil: "folgerichtig". Die Art aber, auf welche Eusebius nach der Erzählung des Märtyrerprozesses IV, I7 noch einmal auf den Crescensabschnitt in IV, I6 zurückkommt, nötigt zu der Folgerung, er habe seine Leser über das unmittelbar Folgende der Erzählung, die er in seiner Quelle fand, belehren wollen. Wenn Eusebius vor Hist. eccl. IV, 17 noch nicht von Crescens gesprochen hätte und dann an die Erzählung des Prozesses unter Urbicus die Worte Justins angeknüpft hätte mit den Worten: hieran knüpft Justin єỉkótwc kai åko$\lambda o u ́ \theta$ we die Voraussagung seines eigenen Todes an, dann brauchte die Anknüpfung keine unmittelbare zu sein. Jetzt aber verhält sich die Sache ganz anders. Warum kommt Eusebius am Ende von IV, 17 noch einmal auf den Crescensabschnitt IV, 16 zurück? Weil seine Leser durch die Reihenfolge, in welcher er erst über Crescens und dann über Urbicus spricht, leicht auf den Gedanken hätte kommen können, der Bericht über Crescens stehe in Justins Apologie vor der Erzählung des Märtyrerprozesses. Er will diesen möglichen Irrtum zurückhalten. Es ist ihm alles daran gelegen, seinen Lesern die Wahrheit mitzuteilen. Daß Eusebius ein so geriebener Schriftsteller gewesen sein soll, der jene feinen Mittel kannte, durch die der Autor den Geschichtsschreiber und die Leser ohne Aufsehen zu täuschen vermag ${ }^{4}$, habe ich, wenigstens Justin gegenüber, niemals verspürt. Hier ist er sogar peinlich genau! Man merke wohl, daß Eusebius die Worte Justins "buchstäblich" anzuführen anfängt, mit folgendem u. s. w. Damit hat er nichts andres sagen

x Überweg (Grundriß d. Gesch. d. Philos., II, ed. 2, S. $3^{8}$ ) will sogar die ganze kaiserliche Familie erwähnt haben!

2 T. u. U. I, S. $139 . \quad 3$ A. a. O. S. I19. \& Harnack, T. U. U. I, S. 14I. 
wollen, als was jedermann mit einer solchen Bemerkung sagt. „Auf diese Erzählung läßt Justin schicklich und folgerichtig die oben von uns erwähnten Worte folgen: Auch ich erwarte nun, u. s. w." Welcher vorurteilsfreie Mensch würde diese Bemerkung anders auffassen, als daß die Worte: Auch ich erwarte nun, u. s. w. „unmittelbar" auf diese Erzählung folgten? Und wenn in den MSS. zwischen dieser Erzählung und den Worten: Auch ich erwarte nun u. s. w. nicht fünf Kapitel stünden, würde es keinem in den Sinn kommen, sie anders aufzufassen.

Harnack und auch Veil meinen, daß der Zusammenhang der Rede gestört wird, wenn man c. 3 zwischen c. 2 und c. 4 einschiebt. C. 3 ..

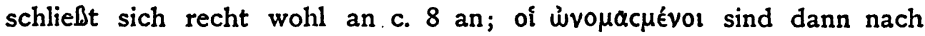
Harnack die Dämonen; nach Veil die Dämonendiener, und c. 9 init.

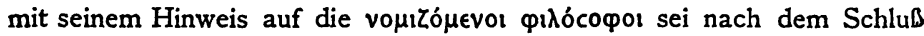
der auf den Cyniker bezüglichen Ausführungen nichts weniger als befremdlich. Hört man dagegen Maranus, den v. Otto mit Zustimmung anführt, ${ }^{x}$ dann bekommt man einen ganz anderen Ëindruck: Totus hic de Crescente locus quum. percommode (sic!) cum martyrum historia

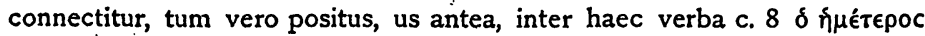

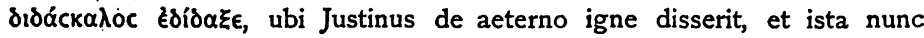

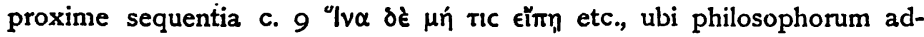
versus ignem aeternum cavillationes refellit, sententiae seriem, ut quisque facile perspicit, mirum in modum turbaret (!) Ich gebe Maranus und v. Otto unbedingt Recht. C. 3, das nicht mit 'Erú, sondern mit Kárú anfängt, kann, weil darin von einem Märtyrertum die Rede ist, nur an eine Märtyrergeschichte anknüpfen. Auch wird das Argument, daß der

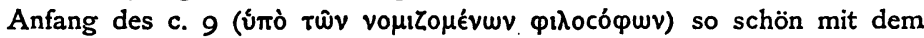
Schluß des c. 5 übereinstimmt (Kuvıkw) hinfällig, wenn man zugibt, daß

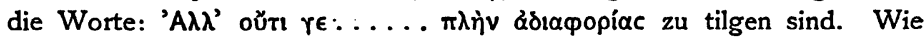
wir wissen, hat Eusebius diese Worte nicht. Warum hat er sie ausgelassen? Er pflegt sonst nicht nachlässig zu zitieren. H. e. II, I3 gibt er den Abschnitt über Simon Magus genau wieder. Ebenso das Zitat über Menander, H. e. III, 26 und Marcion H. e. IV, II. Nur hat

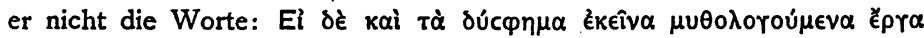
$\pi \rho \alpha ́ \tau t o v a . . . . . e ́ \pi ı c \tau \alpha \mu \epsilon \theta \alpha$. Diese Worte machen den Eindruck einer ungeschickten Interpolation. Warum hat Justin die Ketzerbestreitung überhaupt seiner Apologie eingefügt? hat man gefragt. Weil er die Christen von dem Vorwurf reinigen wolle, sie führten das unsittliche

I Opp. Just. Ed. III, I, I. p. 203, annot. I. 
Leben der Ketzer. Aber diese Antwort genügt durchaus nicht. Hier sagt Justin nachdrücklich: er wisse nichts von solchem unsittlichen Leben der Ketzer! Auch sagt Eusebius nicht, dab er das Zitat tiber Marcion

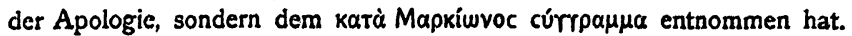
Frühcr war ich der Meinung, Eusebius habe das Zitat der (ersten) Apologie entnommen.t Je mehr ich aber die Genauigkeit des Eusebius zu schätzen anfange, desto mehr komme ich davon zurück, den Eusebius etwas andres sagen zu lassen, als er sagt. Weil man immer wieder seine eigenen voreingenommenen Meinungen dem Eusebius aufbürdet, macht man sich die Sache viel schwieriger und verworrener, als sie wirklich ist. In dem cúrrpa $\mu \mu \alpha$ katà Mapkíwvoc hat er die Stelle über Marcion gelesen, die wir jetzt in der Apologie haben. Toútoıc Ėıৎє́pєl

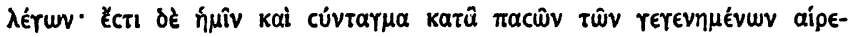
cewv .. T. $\lambda$. So schliellt Eusebius sein Zitat. Man kann diese Worte doch schwerlich anders verstehen, als daß die Bemerkung über das Syntagma gegen all die bisher aufgetretenen Irrlehren sich in der Quelle des Eusebius "unmittelbar" an die Marcionstelle anschlo $\emptyset$, und weiter,

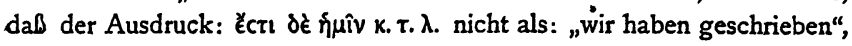

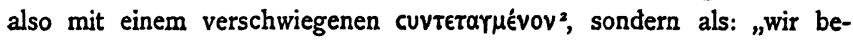
sitzen" aufzufassen ist.

I Theol. Studiën IX, $189 \mathrm{I}$, Bl. 324 v. 0 .

2 So v. Otto. Opp. Just. Ed. III, I, I p. 84, annot. 33. Auch Veil, a, a. O. S. I7.

[Din Schlussartikel folgt.] 